AUS DEM LEHRSTUHL

FÜR PHYSIOLOGIE

PROF. DR. A. KURTZ

NATURWISSENSCHAFTLICHE FAKULTÄT III

BIOLOGIE \& VORKLINISCHE MEDIZIN

DER UNIVERSITÄT REGENSBURG

\title{
NATRIURETIC PEPTIDES BUFFER RENIN-DEPENDENT HYPERTENSION
}

\author{
Inaugural - Dissertation \\ zur Erlangung des Doktorgrades \\ der Medizin \\ der Fakultät für Medizin \\ der Universität Regensburg
}

\author{
vorgelegt von \\ Theo Demerath \\ aus Trier
}



AUS DEM LEHRSTUHL

FÜR PHYSIOLOGIE

PROF. DR. A. KURTZ

NATURWISSENSCHAFTLICHE FAKULTÄT III

BIOLOGIE \& VORKLINISCHE MEDIZIN

DER UNIVERSITÄT REGENSBURG

\title{
NATRIURETIC PEPTIDES BUFFER RENIN-DEPENDENT HYPERTENSION
}

\author{
Inaugural - Dissertation \\ zur Erlangung des Doktorgrades \\ der Medizin \\ der Fakultät für Medizin \\ der Universität Regensburg
}

\author{
vorgelegt von \\ Theo Demerath \\ aus Trier
}


Dekan:

1. Berichterstatter:

2. Berichterstatter:

Tag der mündlichen Prüfung:
Prof. Dr. Dr. Torsten E. Reichert

Prof. Dr. Frank Schweda

Prof. Dr. Matthias Mack

18. Mai 2015 


\section{Zusammenfassung}

Natriuretic peptides buffer renin-dependent hypertension. Theo Demerath, Janina Staffel, Andrea Schreiber, Daniela Valletta, Frank Schweda: Institut für Physiologie, Universität Regensburg. Am J Physiol Renal Physiol. 306: 1489-1498, 2014.

http://www.ncbi.nlm.nih.gov/pubmed/24717731

\section{Fragestellung}

Das Renin-Angiotensin-Aldosteron-System (RAAS) hat eine bedeutende Funktion bei der Regulation der Salz- und Wasserhomöostase des Körpers sowie des Blutdrucks. Geschwindigkeitsbestimmend ist in der RAAS-Kaskade die Aktivität der Aspartylprotease Renin, die aus den juxtaglomerulären Zellen der Niere freigesetzt wird. Renin spaltet nach seiner Freisetzung in den Blutkreislauf von dem in der Leber gebildeten Angiotensinogen das Dekapeptid Angiotensin I ab. Durch die Abspaltung von zwei weiteren Aminosäuren durch das Angiotensin-Converting-Enzym (ACE) entsteht aus Angiotensin I das biologisch aktive Angiotensin II. Angiotensin II ist der Haupteffektor des RAAS und wirkt zum einen über eine Vasokonstriktion blutdrucksteigernd. Darüberhinaus stimuliert Angiotensin II an der Nebennierenrinde die Freisetzung von Aldosteron und steigert dadurch die NaCl-Retention in der Niere (7). Dieser indirekte renale Effekt ergänzt den direkten vasokonstriktorischen Effekt an den Blutgefäßen und wirkt ebenfalls blutdrucksteigernd. Neben seinem blutdrucksteigernden Effekt und zahlreichen weiteren Wirkungen im Körper hemmt Angiotensin II in Sinne einer negativen Rückkopplung auch direkt die Reninsekretion.

Die Freisetzung von Renin unterliegt darüberhinaus einer komplexen Regulation durch eine Vielzahl von lokalen und systemischen Faktoren. Auf systemischer Ebene wirken dabei drei Hauptkontrollfaktoren auf die Synthese und Freisetzung von Renin $(5,23)$ :

1. Die Kochsalzaufnahme und der Kochsalzbestand des Körpers

2. Das sympathische Nervensystem

3. Der renale Perfusionsdruck

Pathophysiolgisch kommt dabei dem renalen Perfusionsdruck eine besondere Bedeutung zu. So führt eine einseitige Nierenarterienstenose zu einem Abfall des renalen Perfusionsdrucks distal der Stenose. Da eine inverse Beziehung zwischen renalem Perfusionsdruck und renaler 
Renin-Freisetzung besteht, kommt es zu einer Stimulation der Reninsynthese und -freisetzung in/aus der betroffenen Niere und dadurch zu erhöhten Renin und Angiotensin II Spiegeln im Blut. Durch die blutdrucksteigernde Wirkung von Angiotensin II entwickelt sich daher eine Renin-abhängige renovaskuläre arterielle Hypertonie.

Die Funktion des RAAS bei der Regulation von extrazellulärem Blutvolumen und Blutdruck wird von den natriuretischen Peptiden ANP (atriales natriuretisches Peptid) und BNP (B-Typ natriuretisches Peptid) antagonisiert. Beide Peptide werden vorwiegend aus dem Herz sezerniert und stellen somit eine hormonelle Verbindung zwischen Herz und Nieren dar (3, 15, 17). Der Hauptstimulus für die Sekretion von ANP und BNP in den Blutkreislauf ist eine Zunahme der Wandspannung im Herzen als Folge einer Druck- oder Volumenbelastung. Da die natriuretischen Peptide u.a. die Diurese und Natriurese steigern und zudem direkt vasorelaxierend wirken, besitzen sie blutdrucksenkende Eigenschaften. Darüber hinaus hemmt ANP direkt die Renin-Freisetzung aus juxtaglomerulären Zellen (11) und ANP und BNP können die Plasma-Renin-Aktivität (PRA) in vivo herabsetzen (1, 8, 23). Bisher bekannte Zielstrukturen der natriuretischen Peptide ANP und BNP sind die Natriuretischen Peptid Rezeptoren-A und C (NPR-A, NPR-C). Der NPR-A ist eine membranständige Guanylatzyklase (GC-A) und seine Aktivierung führt folglich zu cGMP-Bildung. Der Rezeptor NPR-C dient durch Rezeptor-induzierte Internalisation und Degradation von natriuretischen Peptiden vorwiegend als „Clearance-Rezeptor“.

Die Plasmakonzentrationen von Renin und den natriuretischen Peptide stehen unter den physiologischen Bedingungen in einer inversen Beziehung. So sind beispielsweise die Plasmakonzentrationen von ANP und BNP unter hoher Salzaufnahme und Hypervolämie erhöht, wohingegen die PRA in diesen Fällen supprimiert ist $(2,13,16)$. Diese inverse Beziehung gilt nicht in pathologischen Zuständen wie der Herzinsuffizienz, in der sowohl die natriuretischen Peptide als auch das RAAS hochreguliert sind. Da die genetische Deletion oder die pharmakologische Blockade der GC-A zu einem deutlichen Anstieg der Plasma-ReninKonzentration (PRC) bei herzinsuffizienten Mäusen führt $(18,28)$, scheinen die natriuretischen Peptide somit den Anstieg der Reninaktivität bei Herzinsuffizienz abzupuffern. Auch bei der Renin-abhängigen renovaskulären Hypertonie, der häufigsten Form der sekundären Hypertonien sind sowohl Renin-, als auch ANP-Plasmaspiegel erhöht (4, 12, 27, 30) und pharmakologische Dosen von ANP führen zu einer Suppression der Plasma-Renin-Aktivität (6). Ob allerdings auch der Anstieg von endogenem ANP als Folge der Nierenarterienstenose den begleitenden Anstieg der Reninfreisetzung und damit den Blutdruckanstieg abschwächt, ist 
nicht bekannt. Um diese Frage zu beantworten und um die Interaktion zwischen den natriuretischen Peptiden und dem RAAS weiter aufzuklären, wurden Experimente an ANP und GC-A Knockout Mäusen unter Anwendung der experimentellen Nierenarterienstenose durchgeführt.

\section{Methoden}

Versuchstiere: Es wurden männliche, 16-24 Wochen alte ANP-Knockout Mäuse (in C57BL/6 Hintergrund eingekreuzt) und Guanylatzyklase-A Knockout Mäuse (SV129/C57BL/6 Hintergrund) verwendet. Zur Vermeidung von Effekten, die auf dem genetischen Hintergrund basieren, wurden in der Studie die Nachkommen von heterozygoten Zuchtpaaren verwendet. Für die Versuche an der isoliert perfundierten Niere wurden C57BL/6-Mäuse erworben (Charles River, Deutschland).

Unilaterale Nierenarterienstenose: Die unilaterale Nierenarterienstenose (2-kidney, 1-clip, $2 \mathrm{k} 1 \mathrm{c}$ ) wurde wie vorbeschrieben (29) durchgeführt. Vor dem Eingriff wurden der wachen Maus $20 \mu \mathrm{L}$ Blut aus der V. facilialis entnommen, um den Basiswert der PRC zu bestimmen. Danach wurden die Mäuse mit Sevoflurane betäubt, die linke Niere wurde durch eine kleine Inzision freigelegt, die Nierenarterie wurde von der Nierenvene isoliert und ein U-förmiger Silber-Clip (mit einem inneren Durchmesser von 0,11 mm) wurde um die Nierenarterie gelegt. Die gleiche chirurgische Prozedur, mit Ausnahme der Anwendung des Clips wurde an der „sham“Kontrollgruppe an Mäusen durchgeführt. Sieben Tage nach Clipping wurden Blut (PRC und Plasma-ANP und -BNP-Konzentrationen) und Organe entnommen. Die Organe wurden gewogen, schockgefroren und für das weitere Vorgehen bei $-80^{\circ} \mathrm{C}$ gelagert.

Blutdruckmessungen Mittels „,Tail cuff Methode“: Die Mäuse wurden vor der ersten Messung für 7 Tage in den Halteapparaten konditioniert. Pro Tag wurden je Maus 8 Blutdruckwerte sowie die Herzfrequenz bestimmt und es wurde der Durchschnittswert von 5 aufeinander folgenden Tagen vor $2 \mathrm{k} 1 \mathrm{c}$ ermittelt. Es wurden zudem die Blutdruckwerte sowie Herzfrequenz an den Tagen 3 bis 7 nach Nierenarterienstenose bestimmt. Die Durchschnittswerte der täglichen Blutdruckwerde von jedem Tier wurden von Tag 4-7 für die Analyse berechnet.

Bestimmung der Plasma ANP und BNP Konzentrationen sowie der Plasma Renin Konzentration (PRC): ANP und BNP wurden unter Anwendung der entsprechenden 
Enzymimmunoassays (Phoenix Pharmaceuticals Inc.) bestimmt. Die Messung der PRC erfolgte mittels Radioimmunoassay (Dia Sorin).

Bestimmung des Plasmavolumens (durchgeführt von Janina Staffel, Institut für Physiologie, Universität Regensburg): Das Plasmavolumen wurde über die Evans Blue Dilutionsmethode

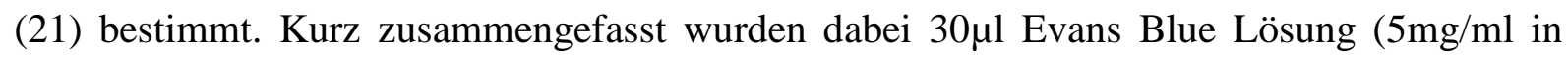
steriler Salzlösung) retrobulbär unter leichter Anästhesie (Sevofluran) injiziert. Blutproben wurden aus der Schwanzvene vor (Baseline) und 10-30 Minuten nach Injektion von Evans Blue entnommen. Nach Zentrifugation wurde die Absorption bei $620 \mathrm{~nm}$ bestimmt und die Evans Blue Konzentration wurde anhand einer Standardkurve berechnet. Das Plasmavolumen wurde nach einem linearen Regressionsmodell berechnet.

Bestimmung der mRNA Expression durch real-time PCR: Die Gesamt-RNA wurde aus den gefrorenen Nieren Mittels TRIzol Reagenz isoliert. Nach der Reversen Transkription wurde die real-time RT-PCR durchgeführt, um Mittels LightCycler die Renin- und B-Aktin-Expression zu ermitteln.

Immunofluoreszenz von Renin: Die Nieren wurden durch Perfusion mit Paraformaldehyd (4\%) fixiert, in Paraffin eingebettet und in $5 \mu \mathrm{m}$ Schichten geschnitten. Die Färbungen wurden nach einem bekannten Verfahren (22) unter Anwendung von direkten Antikörpern gegen Prorenin und Renin durchgeführt.

Isoliert perfundierte Niere der Maus (durchgeführt von Robert Götz, Institut für Physiologie, Universität Regensburg): Nieren von C57BL/6 Mäusen oder GC-A Knockout Mäusen wurden ex situ unter konstantem Perfusionsdruck (100 mmHg) wie im Detail vorbeschrieben (28) perfundiert. Das Perfusionsmedium bestand aus einem modifizierten Krebs-Henseleit-Puffer, der durch Rinder-Serumalbumin $(6 \mathrm{~g} / 100 \mathrm{ml})$ und humane Erythrozyten $(10 \% \mathrm{Hkt})$ ergänzt wurde. Die Nierenvene wurde kanüliert und Proben des venösen Perfusates wurden alle 2 Minuten entnommen, um den renalen Blutfluss und die Plasma-Renin-Konzentration zu bestimmen. Zur Bestimmung der druckabhängigen Regulation der Reninsekretion aus den Nieren von GC-A Knockout Mäusen wurde die Reninsekretion mit dem ß-Adrenorezeptor Agonist Isoproterenol (3mmol/L) vorstimuliert und der Perfusionsdruck wurde stufenweise von 40 auf $140 \mathrm{mmHG}$ verändert.

Statistik: Die präsentierten Werte entsprechen Mittelwerten \pm SEM. Die Unterschiede zwischen den Gruppen oder verschiedenen Zeitpunkten innerhalb einer Gruppe wurden mittels ANOVA 
analysiert und wenn notwendig durch die Bonferroni-Korrektur für multiple Vergleiche bestimmt. In den Experimenten an der isoliert perfundierten Niere wurden Mittelwerte aus den letzten zwei Werten einer experimentellen Periode zur statistischen Analyse bestimmt. Alle statistischen Analysen wurden Mittels GraphPad Prism Software durchgeführt. Eine Irrtumswahrscheinlichkeit von $\mathrm{p}<0,05$ wurde als statistisch signifikant angesehen.

Erklärung: Alle Tierversuche wurden nach den Guidelines für den Umgang und Gebrauch von Versuchstieren nach dem US National Institutes of Health durchgeführt und wurden durch die Regierung der Oberpfalz genehmigt.

\section{Ergebnisse}

\section{Renovaskuläre Hypertension bei ANP Knockout Mäusen}

Wie erwartet, waren die ANP-Knockout Mäuse $\left(\mathrm{ANP}^{-/}\right)$unter Ruhebedingungen hypertensiv (Abb. 1A) und hatten ein erhöhtes Plasmavolumen (ANP ${ }^{-/-}$Mäuse: $4.10 \pm 0.18 \%$ des Körpergewichtes; $\mathrm{ANP}^{+/+} 3.57 \pm 0.08 \%$ des Körpergewichtes, $\mathrm{p}<0.05, \mathrm{n}=6$ Mäuse/Genotyp). Zudem war die Plasma-Renin-Konzentration (PRC) bei $\mathrm{ANP}^{-/-}$signifikant niedriger als beim Wildtyp $\mathrm{ANP}^{+/+}$(Abb. 1C). Um die Hypothese $\mathrm{zu}$ prüfen, ob in Folge einer renalen Hypoperfusion endogenes ANP den Anstieg der PRC hemmt, wurde bei ANP-Knockout Mäusen eine linksseitige Nierenarterienstenose (2-kidney, 1-clip, 2k1c) durchgeführt. Tatsächlich stieg die Plasma-ANP-Konzentration nach Nierenarterienstenose bei $\mathrm{ANP}^{+/+}$ Mäusen auf ungefähr das Doppelte von „sham“-Tieren an (Abb. 2A). Trotz des Fehlens von ANP bei $\mathrm{ANP}^{-/-}$wurde bei beiden Genotypen durch die Nierenarterienstenose jeweils ein deutlicher, ähnlich ausgeprägter Anstieg des Blutdrucks hervorgerufen (Abb. 1A). Aufgrund des höheren Ausgangsblutdruckwertes bei $\mathrm{ANP}^{-/-}$waren unter Nierenarterienstenose die absoluten systolischen Blutdruckwerte bei $\mathrm{ANP}^{-/-}$Mäusen höher als bei den $\mathrm{ANP}^{+/+}$Mäusen (Abb. 1A). In Relation zum Baseline-Blutdruckwert vor Clipping waren die relativen Blutdruckanstiege bei $\mathrm{ANP}^{+/+}$und $\mathrm{ANP}^{-/}$Mäusen allerdings nahezu identisch. Die PRC stieg in Folge der Nierenarterienstenose bei $\mathrm{ANP}^{+/+}$und $\mathrm{ANP}^{-/-}$deutlich an (Abb. 1C). Während die PRC bei geclippten $\mathrm{ANP}^{+/+}$Mäusen signifikant höher als bei „sham“--ANP ${ }^{+/+}$Mäusen war, zeigte sich bei geclippten $\mathrm{ANP}^{-/-}$Mäusen im Vergleich zur „sham“-Gruppe nur eine (nicht signifikante) Tendenz zu einer erhöhten PRC ( $p=0.1$, Abb. 1C). Die Absolutwerte der PRC waren bei $\mathrm{ANP}^{-/-}$Mäusen nicht nur unter Ruhebedingungen, sondern auch nach 
Nierenarterienclipping signifikant niedriger als bei $\mathrm{ANP}^{+/+}$Mäusen. Die relativen Anstiege der $\mathrm{PRC}$ nach 2k1c zwischen $\mathrm{ANP}^{+/+}$und $\mathrm{ANP}^{-/-}$waren nicht signifikant unterschiedlich. Die renale Renin-mRNA-Expression und der renalen Renin-Gehalt waren bei „sham“-ANP ${ }^{-/-}$Mäusen niedriger als bei „sham“-ANP ${ }^{+/+}$Mäusen (Abb. 3A u. B). Die Renin-Synthese, die durch die Menge an Renin-mRNA und Renin-Gehalt wiedergespiegelt wird, wurde bei beiden Genotypen durch die Nierenarterienstenose in der betroffenen (linken) Niere stimuliert, wobei sie in der kontralateralen Niere supprimiert war (Abb. 3). Die relativen Veränderungen der Reninsynthese waren bei $\mathrm{ANP}^{-/-}$und $\mathrm{ANP}^{+/+}$Mäusen ähnlich. Als wichtiger und unerwarteter Befund zeigte sich, dass die Plasma-BNP-Konzentration zwar bei den „sham“-ANP ${ }^{+/+}$und $\mathrm{ANP}^{-/-}$Mäusen sehr ähnlich war, dass diese jedoch in Folge der Nierenarterienstenose bei ANP /- signifikant anstieg ( $\mathrm{p}=0.007$, Abb. 2B). Die BNP-Spiegel bei $\mathrm{ANP}^{+/+}$Mäusen waren als Folge von 2k1c erhöht, ohne dass eine statistische Signifikanz nachgewiesen werden konnte (p $=0.1$, Abb. 2B). Aufgrund der Tatsache, dass BNP und ANP denselben Rezeptor, die Guanylatcyclase-A (GC-A), aktivieren, erschien es möglich, dass der Anstieg von BNP als Folge der Nierenarterienstenose den Mangel an ANP bei $\mathrm{ANP}^{-/-}$Mäusen kompensieren könnte.

\section{Gesteigerte renovaskuläre Hypertension bei GC-A ${ }^{-/-}$Mäusen}

Um die mögliche Kompensation des Mangels von ANP durch BNP zu verhindern, wurden die 2k1c Versuche an GC-A ${ }^{-/-}$Mäusen und Wildtypen $\left(\mathrm{GC}^{-\mathrm{A}^{+/+}}\right)$wiederholt. Die systolischen Ausgangs-Blutdruckwerte waren bei GC- $\mathrm{A}^{-/-}$höher als bei $\mathrm{GC}-\mathrm{A}^{+/+}$und waren bei beiden Genotypen nach 2k1c im Vergleich zu den Ausgangs-Werten bzw. zu „sham“-Mäusen signifikant erhöht (Abb. 4A). Im Gegensatz zu den Versuchen mit ANP $^{-/-}$Mäusen war der Blutdruckanstieg als Folge des Nierenarterienclipping bei GC-A ${ }^{-/-}$Mäusen etwa doppelt so hoch wie bei GC-A ${ }^{+/+}$. Somit war der relative arterielle Blutdruckanstieg (nach/vor

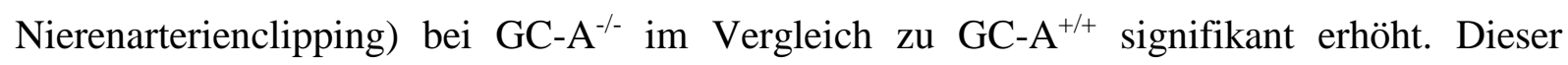
bemerkenswerte Unterschied wird vermutlich noch unterschätzt, da vier GC-A ${ }^{-/-}$Mäuse, die sehr hohe Blutdrücke an Tag 3 nach Nierenarterienclipping aufwiesen, zwischen Tag 3 und 5 verstarben. Diese Mäuse wurden daher aus der Analyse ausgeschlossen. In keiner der anderen Gruppen bzw. des anderen Genotyps starben Mäuse. Nach 2k1c war die PRC bei GC-A ${ }^{+/+}$ Mäusen 10-fach gesteigert, bei GC-A ${ }^{-/-}$nur 2.8-fach, die PRC bei GC-A ${ }^{-/-}$war somit signifikant $(\mathrm{p}<0.001)$ niedriger als bei GC-A $\mathrm{A}^{+/+}(\mathrm{Abb} .4 \mathrm{C})$. In ähnlicher Weise war die Stimulation der Reninsynthese unter Nierenarterienstenose bei GC-A ${ }^{-/-}$Mäusen abgeschwächt, da die renale Renin-mRNA-Expression und der Renin-Gehalt nur bei $2 \mathrm{k} 1 \mathrm{c}$ relativ erhöht schienen, ohne dass eine statistische Signifikanz nachgewiesen werden konnte $(p=0.18$ bzw. $p=0.1$, Abb. 5A u. 
B). Des Weiteren war die Reninsynthese in der unbehandelten rechten Niere bei den geclippten Mäusen im Vergleich zu den "sham"-Mäusen bei beiden Genotypen erwartungsgemäß supprimiert. Die Immunfluoreszenzfärbung von Renin zeigte ein ähnliches Muster (Abb. 6): Bei „sham“-Mäusen färbte sich Renin an den üblichen Regionen Renin produzierender juxtaglomerulärer Zellen bei beiden Genotypen, wobei die gefärbten Areale sowie die Anzahl positiver Zellen bei GC-A ${ }^{-/}$Mäusen kleiner erschienen als bei $\mathrm{GC}^{-\mathrm{A}^{+/+}}$Mäusen. Die Nierenarterienstenose führte bei GC-A ${ }^{+/+}$Mäusen zu einer deutlich verstärkten Renin-Färbung in der geclippten Niere und eine Rekrutierung Renin produzierender Zellen in renalen Blutgefäßen trat auf (s. Beispiel Abb. 6). Während in einigen Glomeruli geclippter Nieren bei GC-A ${ }^{-/-}$Mäusen die Renin-Expression leicht stimuliert wurde, gab es andere, bei denen kein Anstieg der Immunfluoreszenz im Vergleich zu Tieren der „sham“ Gruppe feststellbar war (s. Beispiel Abb. 6).

Das Ausgangs-Plasmavolumen (Abb. 7A, bestimmt durch Janina Staffel, Institut für Physiologie, Universität Regensburg) war bei GC-A ${ }^{-/-}$verglichen mit GC-A ${ }^{+/+}$Mäusen erhöht ( $\mathrm{p}<0.05$ ), zeigte jedoch bei keinem der Genotypen einen weiteren Anstieg in Folge der Nierenarterienstenose bzw. in der „sham“ Gruppe. In der „sham“ Gruppe zeigte sich kein signifikanter Unterschied in der Plasma-ANP-Konzentration zwischen GC-A $\mathrm{A}^{-/-}$und GC-A $\mathrm{A}^{+/+}$ Mäusen, die Anstiege nach 2k1c waren bei beiden Genotypen ähnlich (Abb. 7B). Die BNP Plasmawerte zwischen „sham“ Tieren beider Genotypen unterschieden sich nicht signifikant voneinander. Ähnlich zu dem, was wir bei ANP Knockout Mäusen beobachtet hatten, war der BNP-Spiegel bei GC-A ${ }^{-/-}$in Folge Nierenarterienclipping signifikant erhöht $(\mathrm{p}<0.05)$, wobei bei GC-A $\mathrm{A}^{+/+}$nur ein Trend zu erhöhten BNP Werten feststellbar war $(\mathrm{p}=0.3)$.

\section{Studien an der isoliert perfundierten Niere}

Wie oben gezeigt, war die PRC bei GC-A $\mathrm{A}^{-/-}$Mäusen im Vergleich zum Wildtyp in vivo unter Kontrollbedingungen sowie nach Nierenarterienstenose verringert. Dies könnte entweder durch einen systemischen Phänotyp von GC-A ${ }^{-/-}$Mäusen, der die Reninsynthese hemmt, bedingt sein, oder es könnte eine Folge einer inadäquaten Regulation der Reninsekretion bei Nieren mit fehlender GC-A sein. Um diese Hypothesen zu prüfen, wurden Experimente an isoliert perfundierten Nieren von GC- $\mathrm{A}^{-/-}$und GC- $\mathrm{A}^{+/+}$Mäusen durchgeführt, in denen systemische Einflüsse auf die Reninsekretion wie Hypervolämie oder arterielle Hypertension ausgeschlossen sind (Die Versuchsdurchführung erfolgte durch Robert Götz, Institut für Physiologie, Universität Regensburg). Unter Vorstimulation der Reninsekretionsrate mit 
Isoproterenol ( $3 \mathrm{nmol} / \mathrm{L}$ ) wurde die renale Perfusion auf $40 \mathrm{mmHg}$ reduziert und stufenweise auf 140mmHg angehoben. Die druckabhängige Regulation der Reninfreisetzung war in beiden Genotypen nahezu gleich (Abb. 8A), was eine intakte blutdruckabhängige Regulation der Reninfreisetzung auf Organebene bei GC-A ${ }^{-/-}$Mäusen demonstriert. Darüber hinaus zeigten die GC-A ${ }^{-/-}$Nieren unter Perfusion mit einem Standard- Perfusat, das keine natriuretischen Peptide enthielt keine alterierte Antwort auf ANG II, da sowohl die renale Vasokonstriktion (Abb. 8B) als auch die Inhibition der Reninsekretion (Abb. 8C) unter Zugabe von ANG II keine Unterschiede aufwiesen. Dazu passend war die renale ANG II Typ I (AT1)-Rezeptor-mRNAExpression in beiden Genotypen ähnlich.

Um weitere Einblicke in die Mechanismen zu bekommen, über die natriuretische Peptide die ANG II-abhängige renovaskuläre Hypertension puffern, wurde die renovaskuläre Reaktion auf ANG II bei Fehlen bzw. Vorhandensein einer Kombination von ANP und BNP (Abb. 9D u. E) untersucht. Wie aus den Versuchen aus Abb. 9A zu erwarten, verstärkte die Infusion der natriuretischen Peptide die renale Perfusionsrate. Die schrittweise Infusion von ANG II führte zu einer konzentrationsabhängigen Reduktion der renalen Perfusion. Die renale Perfusion war jedoch bei der jeweiligen ANG II-Konzentration unter Anwesenheit von natriuretischen Peptiden höher als bei Nieren ohne gleichzeitige Zugabe natriuretischer Peptide (Abb. 9D). Selbst in Relation zu den Baseline-Werten (mit/ohne natriuretische Peptide, Abb. 9E), wurde die dosisabhängige Kurve durch natriuretische Peptide nach rechts verschoben, was zeigt, dass die renalen Gefäße bei fehlender Aktivierung der Signalwege der natriuretischen Peptide sensitiver auf ANG II mit Vasokonstriktion reagieren.

\section{Diskussion}

Das Renin-Angiotensin-Aldosteron-System, RAAS, und das Natriuretische-Peptid-System mit den kardialen natriuretischen Peptiden ANP und BNP und dem gemeinsamen GC-A Rezeptor wirken als Antagonisten bei der Blutdruckregulation. Beide Systeme beeinflussen den Blutdruck einerseits indirekt renal über die Kontrolle des Extrazellularvolumens, andererseits durch Wirkungen im Herz-Kreislauf-System, einschließlich direkter Wirkungen am Gefäßsystem. Die von ANP und BNP induzierte Natriurese und Diurese, die Vasorelaxation und die Suppression des Plasma-Renin-Konzentration führen zu einer Blutdruckreduktion (1, 8). Die Inhibition der Reninfreisetzung kann direkt an den Renin produzierenden juxtaglomerulären Zellen (11) erfolgen und könnte indirekte tubuläre und endokrine 
Mechanismen von natriuretischen Peptiden beinhalten (23). Bei der renovaskulären Hypertension wird der Anstieg der Plasma-Renin-Aktivität, welcher den Blutdruckanstieg verursacht, von einer hohen ANP-Plasma Konzentration begleitet (4, 12, 27, 30). Es erscheint daher plausibel, dass diese hohen ANP- Konzentrationen die Hochregulation der Reninsynthese abschwächen und somit die renovaskuläre Hypertension abpuffern könnten.

Unsere Daten zeigen tatsächlich, dass die renale Hypertension durch natriuretische Peptide gepuffert wird. Der Blutdruckanstieg als Folge der Nierenarterienstenose bei fehlenden natriuretischen-Peptid-Signalwegen wird jedoch nicht durch eine Disinhibition der Reninsynthese oder -freisetzung hervorgerufen. Sowohl ANP als auch GC-A Knockout Mäuse waren wie bereits gezeigt $(9,10,14)$ unter Kontrollbedingungen hypertensiv. Bei ANP Wildtyp und GC-A Wildtyp und Knockout Mäusen waren unter Nierenarterienstenose die Plasma-ANPKonzentrationen signifikant erhöht, was an Ratten bereits gezeigt werden konnte (30). Trotz des Fehlens von ANP wurde durch die Nierenarterienstenose ein vergleichbarer Blutdruckanstieg bei $\mathrm{ANP}^{-/-}$und dem Wildtyp hervorgerufen, ohne dass es Hinweise auf eine Deregulation der Reninsynthese und -freisetzung gab. Ein verstärkter Anstieg der PlasmaRenin-Konzentration bei $\mathrm{ANP}^{-/}$, wie man bei einer puffernden Wirkung von ANP auf die Reninfreisetzung erwarten sollte, war nicht nachweisbar. Bestätigt wird diese Schlussfolgerung durch die fehlende Disinhibition der Regulation der renalen Renin-mRNA und des renalen Renin-Gehaltes bei $\mathrm{ANP}^{-/-}$Mäusen.

Wie bereits vorbeschrieben (26) zeigten die $\mathrm{ANP}^{-/-}$Mäuse unter Kontrollbedingungen eine normale Plasma-BNP-Konzentration. An Ratten konnte bereits gezeigt werden, dass BNP innerhalb der ersten zwei Tage nach $2 \mathrm{k} 1 \mathrm{c}$ rasch hochreguliert wird und BNP an Tag 4 wieder Normalwerte erreicht (30). In unserer Studie schien die Plasma-BNP-Konzentration von Wildtyp Mäusen über 7 Tage nach Nierenarterienclipping erhöht zu sein; hingegen war bei den $\mathrm{ANP}^{-/-}$und $\mathrm{GC}^{-\mathrm{A}^{-/-}}$Mäusen das Plasma-BNP nach 2k1c signifikant höher als in den scheinoperierten Mäusen. In Zusammenschau mit der Tatsache, dass ANP und BNP über den GC-A Rezeptor einen gemeinsamen Signalweg besitzen und der nachgewiesenen negativen Wirkung von BNP auf PRC und Blutdruck $(8,19)$, weisen unsere Ergebnisse darauf hin, dass BNP einen Mangel an ANP bei $\mathrm{ANP}^{-/-}$Mäusen möglicherweise kompensiert. Dafür spricht möglicherweise auch, dass vier von acht $\mathrm{GC}^{-\mathrm{A}^{-/}}$in der ersten Woche nach Nierenarterienstenose verstarben. Diese vier Tiere hatten die höchsten Blutdruckwerte aller untersuchten Mäuse. Auch wenn keine detaillierte Autopsie erfolgte erscheint es wahrscheinlich, dass diese Mäuse durch hypertensive Komplikationen verstarben, was erneut 
die Bedeutung der natriuretischen-Peptid-Systems in dieser Situation unterstreicht. Der deutliche Blutdruckanstieg der GC-A ${ }^{-/}$Mäuse war nicht Folge eines überaktivierten Reninsystems. Stattdessen wurde die PRC bei den $\mathrm{GC}^{-A^{-/}}$Mäusen durch das Nierenarterienclipping nur leicht stimuliert und die Stimulation der Reninsynthese in der geclippten Niere, wie sie bei den GC-A ${ }^{+/+}$Mäusen beobachtet werden konnte, war bei den GC$\mathrm{A}^{-/-}$Mäusen deutlich schwächer. Dies weist darauf hin, dass die Stimulation der Reninsynthese unter niedrigem renalen Perfusionsdruck bei GC-A ${ }^{-/-}$Mäusen nicht funktioniert, oder dass andere Faktoren wie die arterielle Hypertension die Reninsekretion hemmen.

Um die erste Hypothese zu überprüfen, wurden Experimente an dem Modell der isoliert perfundierten Niere durchgeführt. Dies ermöglicht die Untersuchung der renalen Reninfreisetzung und der renalen Perfusion unter kontrollierten Bedingungen, beispielsweise ohne den Einfluss systemischer Nebeneffekte. Die inverse Beziehung zwischen Perfusionsdruck und der Reninsekretionsrate zeigte die Intaktheit der druckabhängigen Regulation der Reninfreisetzung bei GC-A ${ }^{-/-}$Mäusen. Darüber hinaus wurde die Reninsekretion bei GC- $\mathrm{A}^{-/-}$Nieren regelgerecht durch den $\beta$-Adrenorezeptoragonisten Isoproterenol stimuliert und durch ANG II inhibiert. Demzufolge ist die verringerte PRC in vivo bei GC-A ${ }^{-/-}$Mäusen sehr wahrscheinlich nicht durch einen intrinsischen renalen Defekt in der Regulation der Reninsekretion bei GC-A ${ }^{-/-}$bedingt, sondern eine indirekte Konsequenz des systemischen GC$\mathrm{A}^{-/-}$Phänotyps. Tatsächlich haben ANP und GC-A Knockout Mäuse verglichen mit dem Wildtyp höhere Plasmavolumina und eine arterielle Hypertension, beides Faktoren, die die Reninsynthese und -freisetzung hemmen.

Sowohl ANP als auch BNP induzierten konzentrationsabhängig eine Vasorelaxation an isoliert perfundierten Nieren; dieser Effekt könnte eine gute Erklärung für die blutdruckabhängige Pufferkapazität der natriuretischen Peptide sein. Zudem führte eine Kombination von ANP und BNP zu einer Abschwächung der Angiotensin II abhängigen Vasokonstriktion an isoliert perfundierten Nieren. Da ANP und BNP über den GC-A Rezeptor wirken, sollte dieser antikontraktile Effekt der natriuretischen Peptide in vivo nicht bei GC-A Knockout Mäusen vorhanden sein und daher die vaskuläre Sensitivität gegenüber Angiotensin II und damit ein Angiotensin II induzierter Blutdruckanstieg bei GC-A ${ }^{-/-}$Mäusen im Vergleich zum Wildtyp verstärkt sein. Andere Faktoren, wie das erhöhte Plasmavolumen, könnten zusammen mit der vaskulären Hypersensitivität erklären, warum der Blutdruck bei GC-A ${ }^{-/-}$Mäusen nach Nierenarterienclipping trotz nur geringer Aktivierung des RAAS so stark ansteigt. Die Rolle der natriuretischen Peptide bei der Blutdruckregulation ist durch genveränderte Tiermodelle 
belegt. Die genetische Überexpression von ANP, BNP und dem GC-A Rezeptor führt ähnlich wie bei pharmakologischer Anhebung der Plasma-ANP- und BNP-Konzentration zu Hypotension (19, 20, 24). Damit vereinbar ist, dass GC-A und ANP Knockout Mäuse hypertensiv sind $(9,10,14)$. Während ANP Knockout Mäuse dem hypertensiven Phänotyp der GC-A Knockout Tiere weitgehend ähneln, führt die BNP-Deletion nicht zu Hypertension (9, 25). Unter physiologischen Bedingungen scheint BNP keine essentielle Rolle bei der Blutdruckregulation zu spielen, es scheint eher, dass BNP in pathologischen Situationen wie der kongestiven Herzinsuffizienz, die zu hohen Plasma-BNP-Konzentrationen führen, bedeutsam ist (17). In unserer Studie waren die Plasma-ANP-Konzentrationen bei Wildtyp Mäusen unter renaler Hypertension signifikant erhöht, was zeigt, dass ANP auch bei ansonsten gesunden Mäusen als Antwort auf einen Blutdruckanstieg freigesetzt wird. Die Plasma-BNPKonzentrationen waren bei $2 \mathrm{k} 1 \mathrm{c}$ Wildtyp Mäusen im Vergleich mit scheinoperierten Tieren gering, jedoch nicht signifikant erhöht, die BNP-Spiegel wurden jedoch durch Clipping bei $\mathrm{ANP}^{-/-}$und $\mathrm{GC}^{-\mathrm{A}^{-/-}}$signifikant stimuliert, wobei diese bereits unter Ruhebedingungen eine arterielle Hypertension und erhöhtes Plasmavolumen aufweisen. Somit zeigen auch unsere Daten, dass BNP insbesondere in schweren pathophysiologischen Zuständen relevant ist, wohingegen ANP in weniger schwerwiegenden Kreislaufsituationen das dominante natriuretische Peptid darstellt. Des Weiteren erlauben unsere Ergebnisse die Schlussfolgerung, dass der Signalweg der natriuretischen Peptide über GC-A einen wichtigen protektiven und Renin-unabhängigen Mechanismus der Pufferung der renovaskulären Hypertension darstellt. 


\section{Referenzen}

1. Brands MW, Freeman RH. Aldosterone and renin inhibition by physiological levels of atrial natriuretic factor. Am J Physiol Regul Integr Comp Physiol 254: R1011-R1016, 1988.

2. Cavallero S, Gonzalez GE, Seropian IM, Cerrudo CS, Matorra F, Morales C, Hertig CM, Puyo AM, Fernandez BE, Gelpi RJ. Ventricular function and natriuretic peptides in sequentially combined models of hypertension. Am J Physiol Heart Circ Physiol 298: H1290-H1299, 2010.

3. de Bold AJ, Borenstein HB, Veress AT, Sonnenberg H. A rapid and potent natriuretic response to intravenous injection of atrial myocardial exract in rats. Life Sci 28: 89-94, 1981.

4. Fenoy FJ, Quesada T, Garcia-Salom M, Romero JC, Salazar FJ. Hemodynamic effects of chronic infusion of rANP in renal hypertensive rats. Am J Physiol Heart Circ Physiol 256: H1393H1398, 1989.

5. Friis UG, Madsen K, Stubbe J, Hansen PB, Svenningsen $P$, Bie P,Skott $O$, Jensen BL. Regulation of renin secretion by renal juxtaglomerular cells. Pflugers Arch 465: 25-37, 2013.

6. Garcia R, Gutkowska J, Cantin M, Thibault G. Renin dependency of the effect of chronically administered atrial natriuretic factor in twokidney, one-clip rats. Hypertension 9: 88-95, 1987.

7. Hackenthal E, Paul M, Ganten D, Taugner R. Morphology, physiology, and molecular biology of renin secretion. Physiol Rev 70: 1067-116, 1990.

8. Jensen KT, Carstens J, Pedersen EB. Effect of BNP on renal hemodynamics, tubular function and vasoactive hormones in humans. Am J Physiol Renal Physiol 274: F63-F72, 1998.

9. John SW, Krege JH, Oliver PM, Hagaman JR, Hodgin JB, Pang SC, Flynn TG, Smithies O. Genetic decreases in atrial natriuretic peptide and salt-sensitive hypertension. Science 267: 679-681, 1995.

10. John SW, Veress AT, Honrath U, Chong CK, Peng L, Smithies O, Sonnenberg H. Blood pressure and fluid-electrolyte balance in mice with reduced or absent ANP. Am J Physiol Regul Integr Comp Physiol 271: R109-R114, 1996.
11. Kurtz A, Della Bruna R, Pfeilschifter J, Taugner R, Bauer C. Atrial natriuretic peptide inhibits renin release from juxtaglomerular cells by a cGMP-mediated process. Proc Natl Acad Sci USA 83: 4769-4773, 1986.

12. Larochelle $\mathbf{P}$, Cusson JR, Gutkowska J, Schiffrin EL, Hamet P, Kuchel O, Genest J, Cantin M. Plasma atrial natriuretic factor concentrations in essential and renovascular hypertension. Br Med J (Clin Res Ed) 294: 1249$1252,1987$.

13. Lee YJ, Shin SJ, Tan MS, Hsieh TJ, Tsai JH. Increased renal atrial natriuretic peptide synthesis in rats with deoxycorticosterone acetate-salt treatment. Am J Physiol Renal Fluid Electrolyte Physiol 271: F779-F789, 1996.

14. Lopez MJ, Wong SK, Kishimoto I, Dubois S, Mach V, Friesen J, Garbers DL, Beuve A. Saltresistant hypertension in mice lacking the guanylyl cyclase-A receptor for atrial natriuretic peptide. Nature 378: 65-68, 1995.

15. Misono KS, Grammer RT, Fukumi H, Inagami T. Rat atrial natriuretic factor: isolation, structure and biological activities of four major peptides. Biochem Biophys Res Commun 123: 444$451,1984$.

16. Morton JJ, Kenyon CJ, Beattie EC. Hormone and electrolyte changes in post-deoxycorticosterone salt hypertension in rats. J Hypertens 8: 1021-1026, 1990.

17. Mukoyama M, Nakao K, Hosoda K, Suga S, Saito Y, Ogawa Y, Shirakami G, Jougasaki M, Obata K, Yasue H, et al. Brain natriuretic peptide as a novel cardiac hormone in humans. Evidence for an exquisite dual natriuretic peptide system, atrial natriuretic peptide and brain natriuretic peptide. $J$ Clin Invest 87: 1402-1412, 1991.

18. Nishikimi T, Hagaman JR, Takahashi N, Kim HS, Matsuoka H, Smithies O, Maeda N. Increased susceptibility to heart failure in response to volume overload in mice lacking natriuretic peptide receptor-A gene. Cardiovasc Res 66: 94-103, 2005.

19. Ogawa $Y$, Itoh H, Tamura N, Suga S, Yoshimasa T, Uehira M, Matsuda S, Shiono S, Nishimoto H, Nakao K. Molecular cloning of the complementary DNA and gene that encode mouse brain natriuretic peptide and generation of transgenic mice that overexpress the brain natriuretic peptide gene. J Clin Invest 93: 1911-1921, 1994. 
20. Oliver PM, John SW, Purdy KE, Kim R, Maeda N, Goy MF, Smithies O. Natriuretic peptide receptor 1 expression influences blood pressures of mice in a dose-dependent manner. Proc Natl Acad Sci USA 95: 2547-2551, 1998.

21. Oppermann M, Gess B, Schweda F, Castrop H. Atrap deficiency increases arterial blood pressure and plasma volume. J Am Soc Nephrol 21: 468-477 2010 .

22. Schmid J, Oelbe M, Saftig P, Schwake M, Schweda F. Parallel regulation of renin and lysosomal integral membrane protein 2 in reninproducing cells: further evidence for a lysosomal nature of renin secretory vesicles. Pflugers Arch 465: 895-905, 2013.

23. Schweda F, Kurtz A. Regulation of renin release by local and systemic factors. Rev Physiol Biochem Pharmacol 161: 1-44, 2011.

24. Steinhelper ME, Cochrane KL, Field LJ. Hypotension in transgenic mice expressing atrial natriuretic factor fusion genes. Hypertension 16: 301-307, 1990.

25. Tamura N, Ogawa Y, Chusho H, Nakamura K, Nakao K, Suda M,Kasahara M, Hashimoto R, Katsuura G, Mukoyama M, Itoh H, Saito Y, Tanaka I, Otani H, Katsuki M. Cardiac fibrosis in mice lacking brain natriuretic peptide. Proc Natl Acad Sci USA 97: 4239-4244, 2000.
26. Tse MY, Watson JD, Sarda IR, Flynn TG, Pang SC. Expression of B-type natriuretic peptide in atrial natriuretic peptide gene disrupted mice. $\mathrm{Mol}$ Cell Biochem 219: 99-105, 2001.

27. Tunny TJ, Gordon RD, Klemm SA, Stowasser M, Finn WL. Renal extraction of atrial natriuretic peptide in unilateral renal artery stenosis. Clin Exp Pharmacol Physiol 21: 211-214, 1994.

28. Wada A, Tsutamoto T, Matsuda Y, Kinoshita M. Cardiorenal and neurohumoral effects of endogenous atrial natriuretic peptide in dogs with severe congestive heart failure using a specific antagonist for guanylate cyclase-coupled receptors. Circulation 89: 2232-2240, 1994.

29. Wagner C, de Wit C, Kurtz L, Grunberger C, Kurtz A, Schweda F. Connexin 40 is essential for the pressure control of renin synthesis and secretion. Circ Res 100: 556-563, 2007.

30. Wolf K, Kurtz A, Pfeifer M, Hocherl K, Riegger GA, Kramer BK. Different regulation of left ventricular ANP, BNP and adrenomedullin mRNA in the two-kidney, one-clip model of renovascular hypertension. Pflugers Arch 442: 212$217,2001$. 


\section{Danksagung}

Zum Ende möchte ich denjenigen danken, die es ermöglicht haben, die Dissertation erfolgreich abzuschließen. Mein besonderer Dank geht an Prof. Dr. med. Frank Schweda, dessen Ideen und Motivationskraft maßgeblich zum Erfolg der Arbeit beigetragen haben. Besonders danken möchte ich auch Prof. Dr. med. Armin Kurtz, der es mir erlaubte in einem angenehmen Arbeitsumfeld über ein solch spannendes Thema zu promovieren. Des Weiteren möchte ich meinen Kollegen der Arbeitsgruppe für ihre Unterstützung danken, besonders hervorzuheben sind Dr. rer. nat. Andrea Schreiber, Janina Staffel, Dr. rer. physiol. Daniela Valletta sowie Katharina Ehm, Robert Götz und Gerda Treuner. Zuletzt gilt mein Dank im Besonderen auch meinen Eltern und Geschwistern, denen ich meine Arbeit widmen möchte. 